\title{
In the monarch butterfly the juvenile hormone effect upon immune response depends on the immune marker and is sex dependent
}

\author{
Guadalupe Villanueva ${ }^{1}$, Humberto Lanz-Mendoza ${ }^{2}$, Salvador Hernández-Martínez ${ }^{2}$ \\ Minerva Sánchez Zavaleta ${ }^{2}$, Javier Manjarrez ${ }^{1}$, José M. Contreras-Garduño ${ }^{3}$, \\ Jorge Contreras-Garduño ${ }^{4^{*}}$

\footnotetext{
${ }^{1}$ Center of Research in Biotic Resources, Autonomous University of Mexico State, Toluca, México

${ }^{2}$ Center of Research in Infectious Diseases, National Institute of Public Health, Cuernavaca, México

${ }^{3}$ Institute of Ecology, National Autonomous University of Mexico, México City, México

${ }^{4}$ Department of Biology, University of Guanajuato, Guanajuato, México;

*Corresponding author: jcont@ecologia.unam.mx
}

Received 4 December 2012; revised 3 January 2013; accepted 11 January 2013

\begin{abstract}
In insects, juvenile hormone $(\mathrm{JH})$ decreases or has any effect upon the phenoloxidase (PO) activity, and favors or decreases the Antimicrobial Peptides (AMPs) expression. Although there is no information about the differential effect of such hormone, two possibilities are that it depends on (a) the immune marker recorded and (b) sexual differences. Here, three commonly used immune markers, Phenoloxidase (PO), hydrogen peroxide $\left(\mathrm{H}_{2} \mathrm{O}_{2}\right)$, and lytic activity, were measured 3, 6 and 24 hours after administration of methoprene ( $\mathrm{JHa}$, an analog of juvenile hormone) in male and female monarch butterflies (Danaus plexippus). At 3 and $6 \mathrm{~h}$ post-JHa administration, the PO activity increased in females but it only increased at $3 \mathrm{~h}$ in males, whereas $\mathrm{H}_{2} \mathrm{O}_{2}$ levels increased only in females at $3 \mathrm{~h}$. For the remaining times the $\mathrm{JHa}$ had a null effect on $\mathrm{PO}$ and $\mathrm{H}_{2} \mathrm{O}_{2}$. On the other hand, the JHa had a null effect for lytic activity in both sexes at 3, 6 and $24 \mathrm{~h}$. To our knowledge, this is the first report of a positive effect of a JHa on $\mathrm{PO}$ and $\mathrm{H}_{2} \mathrm{O}_{2}$ and suggests that this effect is sex dependent.
\end{abstract}

Keywords: Immune Response; Life History; Juvenile Hormone; Reproductive Diapause; Nitric Oxide

\section{INTRODUCTION}

The endocrine system affects physiology, morphology, behavior and life history [1-4]. As an outstanding example in insects, the juvenile hormone $(\mathrm{JH})$ regulates ovarian development, sexual behavior, reproductive diapause, migratory behavior, resistance to stress and longevity [5-9]. However, an aspect that has been scarcely studied is the effect of $\mathrm{JH}$ upon the immune response. For example in Tenebrio molitor [10] and Calopteryx virgo [11], the application of JH III or methoprene (JHa; an analogue of $\mathrm{JH}$ ) respectively, diminished the production of Phenoloxidase (PO). This is a key enzyme for both the humoral (melanization) and cellular (encapsulation) response against pathogens, as well as wound repair and clotting after injury $[12,13]$. In addition, after the administration of $\mathrm{JH}$ to the larvae of Spodoptera littoralis, there was a reduction of the encapsulation response against the parasitoid Microplitis rufiventris [14]. However, in Hetaerina americana, JHa decreased PO production 3 hours after treatment but not after 24 hours [15]. On the other hand, in Drosophila melanogaster, the application of two analogues of $\mathrm{JH}$, methoprene and piriproxiphene, inhibited the expression of the genes that produce antimicrobial peptides (AMPs), one of the main components of the humoral response [16]. This evidence suggests that $\mathrm{JH}$ could affect negatively the overall immune response. However, in a recent work with Bombyx mori, authors found that $\mathrm{JH}$ favors the AMPs expression [17]. Thus, why the response is not always immunosuppressed due to the $\mathrm{JH}$ application?

There are two hypotheses that may explain why the $\mathrm{JH}$ is not a general immune suppressor: a) some but not all immune markers could be affected in the same way [10] and/or b) it affects differentially males and females, and at the same time, their respective immune response levels. On the one hand, the first explanation predicts that controlling for the $\mathrm{JH}$ doses, some immune markers should decrease, while others could be increased or not be 
affected. This is supported because JH decreased PO and melanization, but any effect was observed in lytic activity [10]. As far as we know this is the only study in which more than one immune parameter has been tested and that supports this hypothesis. On the other hand, the second explanation predicts a sex specific effect due to the $\mathrm{JH}$ action, but as far as we know, this explanation has not been tested because some studies have included only males $[10,11]$ or only females [16] in their experimental designs.

We used the monarch butterfly (Danaus plexippus) to test the above hypotheses because a) whereas with most insects the physiological quantities of JH are unknown in vivo [18], in the monarch butterfly these concentrations have been reported for the adult stage [19], and b) monarchs migrate to Mexico in the winter, and during most of their stay males and females are in reproductive arrest (reproductive diapause), which leads to little variation in JH levels [20]. Regarding the immune markers, the $\mathrm{PO}, \mathrm{H}_{2} \mathrm{O}_{2}$ and lysozyme were used. During melanogenesis to combat parasites and pathogens, $\mathrm{PO}$ catalyzes the hydroxylation of tyrosine to L-dihydroxyphenylalanine (L-DOPA) to produce the melanin precursor, indolequinone [21]. Quinoid compounds are potent catalysts of the production of reactive oxygen molecules (ROS) $[21,22]$, and the oxidation of dihydroxyphenylalanine (L-DOPA) can generate superoxide anion and hydrogen peroxide, which are toxic molecules to parasites [22,23]. In addition, hydrogen peroxide $\left(\mathrm{H}_{2} \mathrm{O}_{2}\right)$ increases the AMP expression in insects [24], suggesting that through this mechanism JH could affect the expression of AMPs. Under this scenario, $\mathrm{JH}$ could not only favor or decrease the AMPs expression, but $\mathrm{PO}$ and $\mathrm{H}_{2} \mathrm{O}_{2}$ production. On the other hand, another immune marker could be the lyzosyme because it degrades the peptidoglycan layer, releasing sugars and exposing molecules, which are then recognized by lectins, which gives the insect the ability to recognize and engulf bacteria [25]. Consequently, the aim of the present study was to evaluate 3 molecules that are generally considered immune markers $\left(\mathrm{PO}, \mathrm{H}_{2} \mathrm{O}_{2}\right.$ and lysozyme) in both males and females of the monarch butterfly during reproductive diapause. In addition, 3 times were established after the application of the $\mathrm{JH}$ to avoid the possibility that the positive, negative or null effect was related to the time elapsed between the administration of the JH [15].

\section{MATERIALS AND METHODS}

\subsection{Animals}

Butterflies (300 males and 350 females) were collected in the butterfly sanctuary in Angangeo, Michoacán, Mexico with a net placed below the branches of a tree. The branches were shaken so that the butterflies perched on them would fall onto the net. Butterflies on the ground were not collected, as they could be near death, injured and/or in a state of lethargy, thus with a compromised immune system. The insects were then separated according to gender by observing the last segments of the ventral part of the abdomen, and placed in paper bags sealed with wax for transport to the lab. All animals were collected before the beginning of reproduction (November-December) [26], this means that they were in reproductive diapause.

\subsection{Preparation of $\mathrm{JHa}$ and Experimental Groups}

For the hormone treatment, the JHa was administered to the adult monarch butterflies by using methoprene (Sigma), which is an analogue of JH III. Concentrations of JHa employed to males and females were based on the quantities of JH III reported previously by Lessman et al., [19]. This let us to work under physiological doses of JH, avoiding the use of pharmacological doses [18]. For the preparation of the solution of methoprene, $5 \mathrm{mg}$ of methoprene were dissolved in $500 \mu \mathrm{L}$ of dimethylsulfoxide (DMSO). From this stock solution, dilutions were made in DMSO grade HPLC to obtain the concentration of $0.46 \mathrm{ng} / \mathrm{mL}$ for males and the $0.2 \mathrm{ng} / \mathrm{mL}$ for the females. According with Lessman et al. [19], these are the doses of $\mathrm{JH}$ found in the hemolymph of each sex respectively. DMSO was used because it is not so toxic for the insects than acetone or methanol [27].

Four experimental groups were formed: one group of males (JHM) and another of females (JHF) treated with $\mathrm{JHa}$, and one group of males (CM) and another of females (CF) that was DMSO-treated (control). All groups were injected with $2 \mu \mathrm{L}$ of the corresponding solution (JHa or DMSO) with a Hamilton micro syringe $(10 \mu \mathrm{L})$, in the membranous area anterior to the thorax, next to where the wings are inserted. Although no real immune challenges were carried out in this study, this method of injection favors the immune response activation because its injury and piercing favor immune response to combat bacterial entrance and leads wound repair [13,28,29]. In addition, studies that have used the $\mathrm{JH}$ or its analogues to record the phenoloxidase (PO) production with [11] or without $[10,30]$ immune challenge reported similar results in different species.

The administered solution was allowed to take effect in vivo during 3, 6 or 24 hours, the times chosen to determine the effect of JH on the immune markers. Three and $24 \mathrm{~h}$ were used because it has been reported a negative and null effect, respectively upon PO [15], and $6 \mathrm{~h}$ was used as medium time period. After this time, hemolymph was extracted from each butterfly to register the activity of the enzymes (PO and lysozyme) and the concentration of $\mathrm{H}_{2} \mathrm{O}_{2}$. 


\subsection{Hemolymph Extraction}

Hemolymph was extracted by perfusion as follows: each butterfly was injected with $50 \mu \mathrm{L}$ of Phosphate Buffer Saline (PBS; Sigma, pH 7.4) between the head and the thorax with a micro syringe (Hamilton; $10 \mu \mathrm{L}$ ). After the injection, each butterfly was decapitated to obtain the hemolymph perfused. Approximately, 10 to 15 $\mu \mathrm{L}$ of the hemolymph perfused were obtained with a micropipette (Rainin $10 \mu \mathrm{L}$ ) and deposited in vials of 1.5 $\mathrm{mL}$ (Axigen) previously pre-cooled, and that contained $60 \mu \mathrm{L}$ of PBS protease inhibitors (Sigma).

\subsection{Protein Content and Phenoloxidase}

The commercial BCA protein assay kit (Thermo scientific) was used. As a standard, bovine serum albumin (BSA; $1 \mathrm{mg} / \mathrm{mL}$ ) was used to know the protein content per sample. Samples were incubated at $37^{\circ} \mathrm{C}$ during 15 minutes and recorded with an Enzyme-linked immunosorbent assay reader (ELISA reader: OpsysMR) at 540 $\mathrm{nm}$ [31]. This method of perfusion has the problem that different amount of protein could be obtained between samples, but to override this factor, the results of protein quantity were used to standardize for $80 \mu \mathrm{g} / \mathrm{mL}$ of protein for each sample. In this way, all samples had the same amount of protein before each analyze of immune markers. In the PO analyses, the oxidation of L-dihydroxiphenylalanina (L-DOPA, Sigma) to dopacrome was recorded because this reaction is carried out in the presence of PO, and then, the dopacrome formation is recorded on a spectrophotometer at $490 \mathrm{~nm}$ (OpsysMR). The samples that contained $80 \mu \mathrm{g} / \mathrm{mL}$ of protein were dose-titer with PBS to obtain $50 \mu \mathrm{L}$ of sample/PBS. In addition, $50 \mu \mathrm{L}$ of L-DOPA $(0.004 \mathrm{~g} / \mathrm{mL})$ were included to each sample-PBS mixture. As blanks, only $50 \mu \mathrm{L}$ of PBS and $50 \mu \mathrm{L}$ L-DOPA $(0.004 \mathrm{~g} / \mathrm{mL})$ were used. This procedure was carried out in all the experimental groups and at different times after the $\mathrm{JHa}$ administration $(3,6$ and $24 \mathrm{~h}$ ).

\subsection{Hydrogen Peroxide and Lytic Activity}

The commercial kit hydrogen peroxide (Invitrogen) was used following the manufacturer instructions. The recordings were performed in an ELISA reader (Opsys$\mathrm{MR})$ at $540 \mathrm{~nm}$. To estimate the lytic activity in each well of a microplate of 96 wells, $9 \mathrm{mg}$ of Micrococcus luteus (Sigma) were dissolved in $25 \mathrm{~mL}$ phosphate buffer $0.1 \mathrm{M}\left(\mathrm{K}_{2} \mathrm{HPO}_{4-} \mathrm{KH}_{2} \mathrm{PO}_{4}\right.$ [Cayman Chemical Co]). Ten $\mu \mathrm{L}$ per sample were included in this mixture and immediately, samples were read at $540 \mathrm{~nm}$. Samples were read every minute during ten minutes. As a control, only the phosphate buffer $0.1 \mathrm{M}$ was mixture with the bacterial solution.

\subsection{Statistical Analysis}

General linear models (GLM; ANOVA factorial) were used and Tukey post hoc tested the differences between groups. In the PO and lysozyme lectures data were rank transformed before the analysis as they not normally distributed, and in the case of hydrogen peroxide a Box cox transformation was enough to reach the normal distribution. All analyses were performed with STATISTICA ${ }^{\circledR}$ (version 8.0). Mean \pm standard error (se) is showed.

\section{RESULTS}

\subsection{Phenoloxidase}

The GLM revealed that the effect of JHa on the expression of PO depended on the treatment $(F=23.96$, $P<0.0001$; Figure 1), the time elapsed after administration of the solution (factorial ANOVA: $F=23.96, P<$ 0.0001 ; Figure 1), and the interaction of time* gender $(F$ $=8.63, P<0.0001$; Figure 1). However, no difference was found with respect to gender $(F=0.06, P=0.806$; Figure 1), and the interactions treatment*gender $(F=$ $1.78, P=0.182$; Figure 1), treatment*time $(F=0.83, P$ $=0.435 ;$ Figure 1) and treatment $*$ time $*$ gender $(F=0.42$, $P=0.655$; Figure 1). The post hoc tests indicated that PO levels were greater in the JHa than control females at 3 (Tukey test: $n=56, P<0.05$ ) and 6 (Tukey test: $n=56$, $P=0.01)$ hours, and no significant difference was found at 24 hours (Tukey test: $n=56, P=0.13$ ). The PO levels were greater in the JHa than control males at 3 hours (Tukey test: $n=53, P=0.04$ ), but no significant differences were observed at 6 (Tukey test: $n=42, P=0.56$ ) or 24 hours (Tukey test: $n=59, P=0.54$ ).

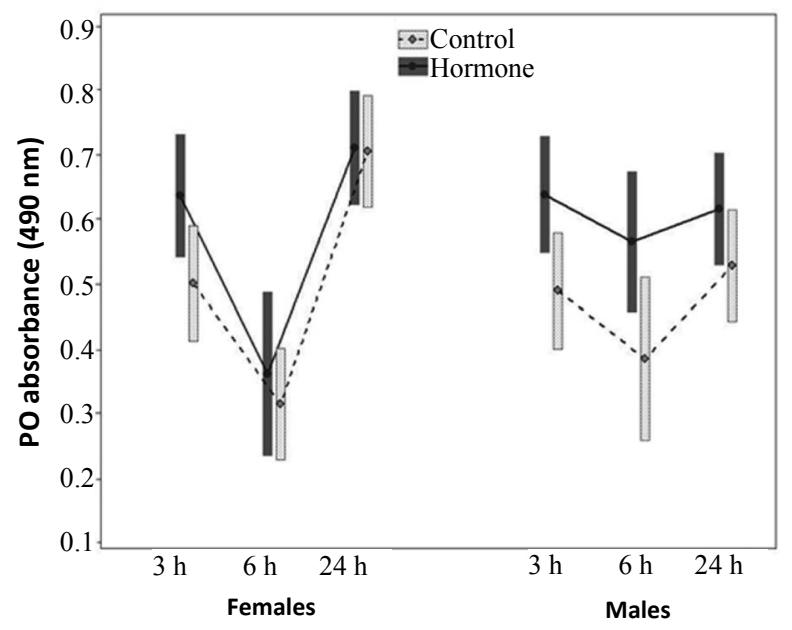

Figure 1. Phenoloxidase from hemolymph of the monarch butterflies at different times (hours), in function of treatment (JH versus control) and gender (females and males). The square represents median and quartiles are represented in bars $(25 \%$ and $75 \%)$. 


\subsection{Hydrogen Peroxide}

The quantity of $\mathrm{H}_{2} \mathrm{O}_{2}$ was related to time $(F=18.37, P$ $<0.01$; Figure 2) and the interaction time*gender $(F=$ $3.73, P=0.02$; Figure 2), but not with the treatment $(F=$ $0.38, P=0.53$; Figure 2), gender $(F=0.39, P=0.53$; Figure 2), or with the interactions treatment*time $(F=$ $0.84, P=0.33$; Figure 2), treatment*gender $(F=2.58, P$ $=0.1$; Figure 2), or treatment*time* gender $(F=0.31, P$ $=0.73$; Figure 2). The post hoc test indicated that the concentration of this compound was greater in the JHa than control females at 3 hours (Tukey test, $n=56, P=$ 0.02 ), but no significant difference was found in the females at 6 (Tukey test, $n=27, P=0.02$ ) and 24 hours (Tukey test, $n=57, P=0.02$ ). On the other hand, neither group of treated males showed any significant difference in the quantity of $\mathrm{H}_{2} \mathrm{O}_{2}$ in respect to the respective control (Tukey test, $n=143, P>0.05$ ).

\subsection{Lytic Activity}

No significant differences were found in the concentrations of lytic activity in respect to treatment $(F=6.50$, $P=0.06)$, gender $(F=0.20, P=0.07)$, or the interactions treatment*time $(F=1.18, P=0.3)$, treatment*gender $(F$ $=0.01, P=0.95)$, or treatment*time* ${ }^{*}$ gender $(F=1.72, P$ $=0.18)$.

\section{DISCUSSION}

This study supports the hypothesis that some, but not all immune markers are affected by the JH. Particularly, it favored $\mathrm{PO}$ and $\mathrm{H}_{2} \mathrm{O}_{2}$ in females but only $\mathrm{PO}$ in males, which also supported the hypothesis that the effect of $\mathrm{JH}$ upon immune response is sex dependent. In addition, we showed that the elapsed time between the JH administration and the immune marker recorded should be taken into account in studies of the effect of JH upon immunocompetence.

In insects, it has been suggested that a single hormone could favor or decrease some but not all immune markers



Figure 2. Concentration of hydrogen peroxide (mean $\pm \mathrm{SE}$ ) from hemolymph at different time points (3, 6 and $24 \mathrm{~h}$ ), according to treatment ( $\mathrm{JH}$ versus control) and gender. at the same time, as for example octopamine (see 4). Rantala et al. [11] previously suggested that some but not all immune markers are affected by the HJ, as they found a decrement in PO and encapsulation but no effect on lytic activity was recorded. Hormones can configure the immune response favoring or affecting it to optimize the response according to different situations [4,32] and this differential effect may reflect changes in immune resource distribution to improve such molecule (s) that is (are) important in a particular situations [33]. Under an evolutionary point of view, could not be adaptive that one molecule ( $\mathrm{JH}$ in this case) may affect the overall immune response at the same time because animals could pay elevated cost of an increased immune response if they are exposed to autoimmune diseases or they could be exposed to parasites and pathogens if all their immune markers are decreased at the same time. Further research is needed to determine the mechanism by which the $\mathrm{JH}$ affects distinct immune markers.

Although it has been suggested that $\mathrm{JH}$ decreases the immune response $[2,10,11,14,16,30]$, in the present study an increase in $\mathrm{PO}$ and $\mathrm{H}_{2} \mathrm{O}_{2}$ was found in females while PO increased in males. This result is in accordance with another study that found that $\mathrm{JH}$ favors antimicrobial peptides (AMPs) [17]. Although the physiological mechanism is still unknown, the reproductive status seems to be implicated. During our study and that of Tian et al. [17] animals were in reproductive diapause or tested as larvae, respectively, while in other studies that reported a negative effect, sexually reproductive animals were used $[10,11]$ supporting the possibility that $\mathrm{JH}$ plays a flexible role upon innate immunity. In a broader sense, it has been proposed that phenomena related to reproduction and development could cause coordinated changes in multiple physiological systems [34,35], as for example, the immune response [36], and this changes may reflect the different trajectories followed by larvae and adults according to particular selective pressures [37]. Clearly, more studies are needed to test if sexual maturity is related with the positive or negative effect of JH upon immune response, the selective pressures related and/or if it could be species dependent [32].

On the other hand, in insects, $\mathrm{JH}$ is expressed in both sexes [34] and differentially affects male and female behavior [38], morphology [39], development and locomotion [38], but the novelty of our results is that the effect of $\mathrm{JH}$ upon immune response seems to be sex dependent at least in the monarch butterfly. In females two out of three immune parameters were affected by the $\mathrm{JH}$ while in males only PO was affected. In addition, the effect on PO remains for longer in females ( 3 and $6 \mathrm{~h}$ ) than males $(3 \mathrm{~h})$. Our results suggest that in monarch butterflies during reproductive diapause, $\mathrm{JH}$ enhanced the expression of immune markers for longer time in females than 
males. Whether these sexual differences are directly related to an enhancement in protection remains to be elucidated but in this case, females could be better protected than males.

\section{CONCLUSION}

This and other studies $[10,15,17]$ suggest that $\mathrm{JH}$ has a positive or any effect on some but not all immune markers, while other studies suggest a negative effect $[10,11$, 14,16,30]. These differential effects could be related with sexual maturity and or reproductive diapause [see also 32] and may impact on the parasite and pathogen resistance. It is fundamental to investigate the mechanism (e.g. molecular or biochemical) that underlies the relationship $\mathrm{JH}$-immune response but taking into account more time points that those used in this study to better describe the kinetics of this relationship.

\section{ACKNOWLEDGEMENTS}

An anonymous reviewer provided substantial comments on the manuscript. Lidia Ruth Ordoñes Espinosa provided help to collect the animals A Grant from CONACyT (152666) was provided to JCG.

\section{REFERENCES}

[1] Nelson, R.J. (2011) An introduction to behavioral endocrinology. 4th Edition, Sinauer Associated Inc., Sunderland.

[2] Flatt, T., Tu, M.P. and Tatar, M. (2005) Hormonal pleiotropy and the juvenile hormone regulation of Drosophila development and life history. Bioessays, 27, 999-1010. doi:10.1002/bies.20290

[3] Zera, A.J., Harshman, L.G. and Williams, T.D. (2007) Evolutionary endocrinology: The developing synthesis between endocrinology and evolutionary genetics. Annual Review of Ecology, Evolution and Systematics, 38, 793817. doi:10.1146/annurev.ecolsys.38.091206.095615

[4] Adamo, S.A. (2009) The impact of physiological state on immune function in insects. In: Reynolds, S. and Rolff, J., Eds., Insect Infection and Immunity, Oxford University Press, Oxford. doi:10.1093/acprof:oso/9780199551354.003.0011

[5] Nijhout, H.F. (1994) Insect hormones. Princeton University Press, Princeton.

[6] Zhou, X.F., Coll, M. and Applebaum, S.W. (2000) Effect of temperature and photoperiod on juvenile hormone biosynthesis and sexual maturation in the cotton bollworm, Helicoverpa armigera: Implications for life history traits. Insect Biochemistry and Molecular Biology, 30, 863-868. doi:10.1016/S0965-1748(00)00059-X

[7] Tatar, M., Chien, S. and Preist, N. (2001) Negligible senescence during reproductive diapause in Drosophila melanogaster. American Naturalist, 158, 248-258. doi:10.1086/321320

[8] Noriega, F.G. (2004) Nutritional regulation of JH synthe- sis: a mechanism to control reproductive maturation in mosquitoes? Insect Biochemistry and Molecular Biology, 34, 687-693. doi:10.1016/i.ibmb.2004.03.021

[9] Hernandez-Martinez, S., Mayoral, J.G., Li, Y. and Noriega, F.G. (2007) Role of Juvenile hormone and allatotropin on nutrient allocation, ovarian development and survivorship in mosquitoes. Journal of Insect Physiology, 53, 230-234. doi:10.1016/j.jinsphys.2006.08.009

[10] Rantala, M.J., Vainikka, A. and Kortet, R. (2003) The role of juvenile hormone in immune function and pheromone production trade-offs: A test of the immunocompetence handicap principle. Proceedings of the Royal Society, 270, 2257-2261. doi:10.1098/rspb.2003.2472

[11] Contreras-Garduño, J., Córdoba-Aguilar, A., Lanz-Mendoza, H. and Cordero, A. (2009) Territorial behaviour and immunity are mediated by juvenile hormone: The physiological basis of honest signaling? Functional Ecology, 23, 157-163. doi:10.1111/j.1365-2435.2008.01485.x

[12] Cerenius, L. and Söderhäll, K. (2004) The prophenoloxidase-activating system in invertebrates. Immunological Reviews, 198, 116-126.

doi:10.1111/j.0105-2896.2004.00116.x

[13] Lemaitre, B. and Hoffmann, J. (2007) The host defense of Drosophila melanogaster. Annual Review of Immunology, 25, 697-743. doi:10.1146/annurev.immunol.25.022106.141615

[14] Khafagi, W.E. and Hegazi, E.M. (2001) Effects of juvenile hormones and precocenes on the immune response of Spodoptera littoralis larvae to supernumerary larvae of the solitary parasitoid, Microplitis rufiventris Kok. Journal of Insect Physiology, 47, 1249-1259. doi:10.1016/S0022-1910(01)00110-X

[15] Contreras-Garduño, J., Villanueva-Guerra, G. and AlonsoSalgado, A. (2012) Phenoloxidase production: The importance of time after juvenile hormone analogue administration in Hetaerina americana Fabricius. Odonatologica, 41, 1-6.

[16] Flatt, T., Heyland, A., Porpiglia, F.R.E., Sherlock, C., Yamamoto, R., Garbuzov, A., Palli, S.R. and Silverman, N. (2008) Hormonal regulation of the humoral innate immune response in Drosophila melanogaster. Journal of Experimental Biology, 211, 2712-2724. doi: $10.1242 /$ jeb. 014878

[17] Tian, 1., Guo, E., Diao, Y., Zhou, S., Peng, Q., Cao, Y., Ling, E. and Li, S. (2010) Genome-wide regulation of innate immunity by juvenile hormone and 20-hydroxyecdysone in the Bombyx fat body. BMC Genomics, 11, 549. doi:10.1186/1471-2164-11-549

[18] Zera, A. (2007) Endocrine analysis in evolutionary-developmental studies of insect polymorphism: Hormone manipulation versus direct measurement of hormonal regulators. Evolution and Development, 9, 499-513. doi:10.1111/j.1525-142X.2007.00181.x

[19] Lessman, C.A., Herman, W.S., Schooley, D.A., Tsai, L.W., Bergot, B.J. and Baker, F.C. (1989) Detection of juvenile hormone I, II and III in adult monarch butterflies (Danaus plexippus). Insect Biochemistry, 19, 431-433. doi:10.1016/0020-1790(89)90049-8

[20] Lessman, C.A. and Herman, W.S. (1983) Seasonal varia- 
tion in hemolymph juvenile hormone of adult monarchs (Danaus p. plexippus: Lepidoptera). Canadian Journal of Zoology, 61, 88-94. doi:10.1139/z83-009

[21] Nappi, A.J. and Vass, E. (1998) Hydrogen peroxide production in immune-reactive Drosophila melanogaster. Journal of Parasitology, 84, 1150-1157. doi: $10.2307 / 3284664$

[22] Nappi, A.J., Vass, E., Frey, F. and Carton, Y. (1995) Superoxide anion generation in Drosophila melanotic encapsulation of parasites. European Journal of Cell Biology, 68, 450-456.

[23] Lanz-Mendoza, H., Hernández-Martínez, S., Ku, M., Gil, A., Rodríguez, M.N. and Rodríguez, M.H. (2002) Superoxide anion in Anopheles albimanus hemolymph and midgut is toxic to Plasmodium berghei ookinetes. Journal of Parasitology, 88, 702-706.

[24] Herrera-Ortiz, A., Martínez-Barnetche, J., Smit, N., Rodriguez, M.H. and Lanz-Mendoza, H. (2011) The effect of Nitric Oxide and Hydrogen Peroxide in the activation of the systemic immune response of Anopheles albimanus infected with Plasmodium berghei. Developmental and Comparative Immunology, 35, 44-50. doi:10.1016/j.dci.2010.08.004

[25] Wilson, R. and Ratcliffe, N.A. (2000) Effect of lysozyme on the lectin-mediated phagocytosis of Bacillus cereus by haemocytes of the cockroach, Blaberus discoidalis. Journal of Insect Physiology, 46, 663-670. doi:10.1016/S0022-1910(99)00154-7

[26] Oberhauser, K.S. and Solensky, M.J. (2004). The monarch butterfly. Biology and conservation. Cornell University Press, Ithaca.

[27] Butcher, F.R. and Perdue, J.F. (1973) Cytochalasina B. Effect on hormone-mediated responses in cultured cells. Journal Cell Biology, 56, 857-861. doi:10.1083/jcb.56.3.857

[28] Wigby, S., Domanitskaya, E.V., Choffat, Y., Kubli, E. and Chapman, T. (2008) The effect of mating on immunity can be masked by experimental piercing in female Drosophila melanogaster. Journal of Insect Physiology, 54, 414-420. doi:10.1016/j.jinsphys.2007.10.010

[29] Rodrigues, J., Brayner, F.A., Alves, L.C., Dixit, R. and Barillas-Mury, C. (2010) Hemocyte differentiation mediates innate immune memory in Anopheles gambiae mos- quitoes. Science, 329, 1353-1355. doi:10.1126/science. 1190689

[30] Rolff, J. and Siva-Jothy, M.T. (2002) Copulation corrupts immunity: A mechanism for a cost of mating in insects. Proceedings of the National Academy of Sciences USA, 99, 9916-9918. doi:10.1073/pnas.152271999

[31] Contreras-Garduño, J., Lanz-Mendoza, H. and CórdobaAguilar, A. (2007) The expression of a sexually selected trait correlates with different immune defense components and survival in males of the American rubyspot. Journal of Insect Physiology, 53, 612-621. doi:10.1016/j.jinsphys.2007.03.003

[32] Adamo, S.A. (2011) The importance of physiology for ecoimmunlogy: Lessons from the insects. In: Demas, G. and Nelson, R., Eds., Ecoimmunology, Oxford University Press, Oxford.

[33] Braude, S., Tang-Martinez, Z. and Taylor, G.T. (1999) Stress, testosterone, and the immunoredistribution hypothesis. Behavioral Ecology, 10, 345-350. doi:10.1093/beheco/10.3.345

[34] Chapman, R.F. (1998) The insects: Structure and function. Cambridge University Press, Cambridge. doi:10.1017/CBO9780511818202

[35] Nation, J. L. (2002) Insect physiology and biochemistry. CRC Press, Boca Raton.

[36] Lawniczak, M.K. N., Barnes, A.I., Linklater, J.R., Boone, J.M., Wigby, S.W. and Chapman, T. (2007) Mating and immunity in invertebrates. Trends in Ecology and Evolution, 22, 48-55. doi:10.1016/i.tree.2006.09.012

[37] Fellous, S. and Lazzaro, B.P. (2011) Potential for evolutionary coupling and decoupling of larval and adult immune gene expression. Molecular Ecology, 20, 15581567. doi:10.1111/j.1365-294X.2011.05006.X

[38] Belgacem, Y.H. and Martin, J.R. (2002) Neuroendocrine control of a sexually dimorphic behavior by a few neurons of the pars intercerebralis in Drosophila. Proceedings of the National Academy of Sciences USA, 99, 15154-15158. doi:10.1073/pnas.232244199

[39] Emlen, D.J. and Nijhout, H.F. (1999) Hormonal control of male horn length dimorphism in the horned beetle Onthophagus taurus. Journal of Insect Physiology, 45, 45-53. doi:10.1016/S0022-1910(98)00096-1 